\title{
Evaluation of carotid intima media thickness and hematologic inflammatory markers in patients with chronic spontaneous urticaria
}

\author{
Mahizer Yaldiz' ${ }^{1}$ Kiyasettin Asil ${ }^{2}$ \\ 1Department of Dermatology, Sakarya Training and Research Hospital, Sakarya, Turkey \\ ${ }^{2}$ Department of Radiology, Sakarya Training and Research Hospital, Sakarya, Turkey
}

Adv Dermatol Allergol 2020; XXXVII (2): 214-220

DOI: https://doi.org/10.5114/ada.2018.79567

\begin{abstract}
Introduction: Low grade inflammation is associated with chronic spontaneous urticaria (CSU) and atherosclerosis. There are little data on the relationship between urticarial inflammation and atherosclerosis. The neutrophil lymphocyte ratio (NLR), platelet lymphocyte ratio (PLR) and mean platelet volume (MPV) have recently been studied in many diseases as a biomarker of inflammation.

Aim: To evaluate hematologic inflammatory parameters and subclinical atherosclerosis in CSU patients.

Material and methods: C-reactive protein (CRP), NLR, PLR, MPV and carotid intima media thickness (CIMT) in CSU patients were compared with healthy controls. Thirty CSU patients and 30 healthy controls participated in the study. Results: Thirty CSU patients and 30 healthy controls participated in the study. $53.3 \%$ of the patient group were females, $46.7 \%$ were males and the mean age was $37.7 \pm 7.7 .53 .3 \%$ of the healthy control group were females, $46.7 \%$ were males and the mean age was $37.1 \pm 7.2$. Body mass index was $24.63 \pm 2.7 \mathrm{~kg} / \mathrm{m}^{2}$ in the patient group and 24.83 $\pm 2.5 \mathrm{~kg} / \mathrm{m}^{2}$ in the control group. Left and right CIMT and CRP values were higher in CSU patients and high-density lipoproteins ( $\mathrm{HDL}$ ) values were lower in the CSU patients. There was a positive correlation between the right and left CIMT and urticaria activity severity 4 (UAS4), disease severity, and triglycerides in the patient group, also a negative correlation was found between the right and left CIMT and the HDL values. C-reactive protein was also correlated with UAS4 and disease severity.

Conclusions: This is the first study to evaluate subclinical atherosclerosis in CSU patients. Chronic spontaneous urticaria may be associated with an increased atherosclerotic risk. Serum CRP concentration can also be used as an objective parameter to evaluate disease severity.
\end{abstract}

Key words: chronic spontaneous urticaria, carotid intima media thickness, hematologic inflammatory markers.

\section{Introduction}

Chronic spontaneous urticaria (CSU) is defined as a common skin disease that is characterized by pruritus and rash that last for more than 6 weeks. Chronic spontaneous urticaria is characterized by nonspecific local inflammation accompanied by systemic acute phase response [1-3].

Inflammation is a process that has an important role in the initiation and progression of atherosclerosis and circulatory diseases [4]. Recently, inflammation has been identified as an independent risk factor for cardiovascular disease [5]. The relationship between an increased C-reactive protein (CRP) level, neutrophil activation, chro- nic systemic inflammation and cardiovascular disease risk has been reported in the literature [6,7].

C-reactive protein is a well-known inflammatory biomarker and increased serum levels have been demonstrated in CSU patients [1]. In addition, it has been shown in the studies that levels of acute phase response (APR)associated inflammatory markers such as CRP, interleukin 6 (IL-6), tumor necrosis factor $\alpha$ (TNF- $\alpha$ ) and matrix metalloproteinase 9 in the circulation increase in CSU. In addition, good correlations of these inflammatory markers with urticaria activity and severity score have been reported [1, 8-10].

In recent years, it has been shown that the neutrophil lymphocyte rate (NLR) and platelet lymphocyte rate (PLR)

\footnotetext{
Address for correspondence: Mahizer Yaldiz, Department of Dermatology, Sakarya Training and Research Hospital, 54098 Sakarya, Turkey, phone: +90 5053572222, e-mail: drmahizer@gmail.com Received: 9.10.2018, accepted: 15.10.2018. 
which are the ratio of neutrophil and platelet counts to lymphocyte counts, are indicative of systemic inflammation and are associated with prognosis in many cardiovascular diseases, malignancies and chronic inflammatory diseases [1, 11-13]. Mean platelet volume (MPV) is considered to be a marker of platelet function and activation, and high MPV values have been shown to be independent risk factors for acute myocardial infarction. In addition, it has been reported that MPV value is high in some systemic inflammatory diseases and has a positive correlation with CRP [14].

In recent years, the emphasis has been laid on the measurement of carotid artery intima media thickness (CIMT) with carotid artery ultrasound in the evaluation of subclinical atherosclerosis. A CIMT increase is considered to be a marker of atherosclerosis [15]. It was first identified in 1986 by Pignoli et al. and there are many literature studies available today [16, 17].

Chronic spontaneous urticaria patients have been reported to have a significantly higher prevalence of the metabolic syndrome when compared to the age-matched control group [18]. The existence of metabolic syndrome often increases the cardiovascular disease risk. Studies show that diseases with chronic inflammation (psoriasis, psoriatic arthritis, Behçet's disease, etc.) pose a risk in terms of atherosclerosis [19].

\section{Aim}

There are little data on the relationship between urticarial inflammation, atherosclerosis and the cardiovascular disease risk. The aim of our study is to examine the hematologic inflammatory marker (NLR, PLR, MPV) and CRP levels in patients with CSU and to evaluate the risk of subclinical atherosclerosis by measuring the thickness of carotid intima media and to determine the correlation of these findings with disease severity and duration.

\section{Material and methods}

\section{Patients}

Thirty CSU volunteer patients (15 ASST+, 15 ASST-) who were followed-up in the chronic urticaria polyclinic of the Ministry of Health Sakarya University Training and Research Hospital and who met the inclusion criteria, and 30 healthy controls who came to the dermatology clinic for causes other than chronic inflammatory diseases and who have not had an urticaria attack before they were enrolled in the study. A local ethics committee approval was obtained before the study (SÜEK.16214662/05.01.04/82).

Patients and controls with classical cardiovascular risks were excluded from the study. In addition, so were those who have a history of cardiovascular, cerebrovascular and peripheral artery disease, collagen tissue disease, systemic vasculitis, autoimmune diseases, thyroid disease, systemic disease and malignancy, those who receive antihyperlipidemic, antihypertensive, antiaggregant, anticoagulant, nitrate, estrogen and oral contraceptive treatment, those who smoke and use alcohol, pregnant women, those who are $<18$ years of age and $>50$ years of age. Cardiovascular risk factors include hypertension (systolic blood pressure $>140 \mathrm{~mm} \mathrm{Hg}$, diastolic blood pressure $>90 \mathrm{~mm} \mathrm{Hg}$, diabetes mellitus (fasting blood glucose: $>110 \mathrm{mg} / \mathrm{dl}$ ), hyperlipidemia (total cholesterol > $240 \mathrm{mg} / \mathrm{dl}$ and/or TG > $160 \mathrm{mg} / \mathrm{dl}$ ), serum creatinine value $>1.3 \mathrm{mg} / \mathrm{dl}$ and obesity (body mass index $>30 \mathrm{~kg} / \mathrm{m}^{2}$ ).

\section{Evaluation of disease severity}

Urticaria activity score (UAS) was evaluated on the day of blood-letting (rash number: no rash: 0, 1-10 wheals $=1,11-50$ wheals $=2$, > 50 wheals $=3+$ pruritus intensity: none: 0 , mild: 1 , moderate: 2 , severe: 3 ; minimum $=0$, maximum $=24)$. UAS was graded as mild (1-8), moderate (9-16) and severe (17-24).

\section{Autologous serum test}

The autologous serum test was applied only to the patient group on the sample blood-letting day. $0.05 \mathrm{ml}$ of autologous serum was given to the inner side of the forearm. Similarly, sterile saline was applied at the same dose as negative control. Thirty minutes after the application, an erythematous urticarial papule formation of $1.5 \mathrm{~mm}$ or greater from negative control was positively evaluated.

Detailed anamnesis-taking and physical examination of all patients were performed. Venous blood samples were taken for hemogram, CRP, total cholesterol, triglyceride, high-density lipoproteins (HDL), low-density lipoproteins (LDL), fasting blood sugar, urea, creatinine, aspartate aminotransferase (AST), and alanine aminotransferase (ALT) determination.

\section{Carotid artery Doppler US evaluation}

Intima media thickness (IMT) measurement software was used by the same radiologist for the measurement of carotid artery intima media thickness of all subjects included in the study with high resolution color Doppler ultrasonography (Toshiba aplio 400, Otawara-shi, Japan) with PLT-1204 BX transducer (7.2-14 MHz). Intima media thickness was measured from CCA, Bulb and ICA levels on both sides and carotid intima media thickness was calculated by the device.

\section{Statistical analysis}

Statistical analyses were evaluated using the Statistical Package for Social Sciences for Windows version 17.0 (SPSS, Chicago, IL, USA) program. Descriptive data were expressed as mean \pm standard deviation, numbers and percentages. Student's t-test was used to compare continuous data. $\chi^{2}$ test was used to compare categori- 
cal data. The relationship between continuous data was evaluated by Pearson correlation test.

$P<0.05$ value was considered as the level of significance. Negative values show an inverse correlation and positive values show a positive correlation in the correlation evaluation.

\section{Results}

Thirty CSU patients and 30 healthy controls participated in the study. $53.3 \%$ of the patient group were females, $46.7 \%$ were males and the mean age was $37.7 \pm 7.7$. $53.3 \%$ of the healthy control group were females, $46.7 \%$ were males and the mean age was $37.1 \pm 7.2$. Body mass index (BMI) was $24.63 \pm 2.7 \mathrm{~kg} / \mathrm{m}^{2}$ in the patient group and $24.83 \pm 2.5 \mathrm{~kg} / \mathrm{m}^{2}$ in the control group. The mean disease duration of chronic urticaria patients was determined as $40.24 \pm 35.36$ months. The average UAS was 16.87 \pm 4.0 . There was no statistically significant difference in terms of age, sex and BMI distribution in both groups (Table 1).
The HDL values were found to be significantly lower in the CSU group as compared to healthy controls, but there was no significant difference between the two groups in terms of LDL, total cholesterol and triglicerydes (TG) values. The CRP level was significantly higher in the CSU group as compared to the control group, but there was no significant difference between two groups in terms of MPV, platelets (PLT), NLR, and PLR. Right and left CIMT scores were $0.54 \pm 0.08,0.53 \pm 0.1$ in the CSU group and $0.48 \pm 0.07,0.46 \pm 0.09$ in the control group, respectively. Right and left CIMT scores were found to be statistically significantly increased in patients with CSU compared to healthy controls (Table 2). There was no correlation between right and left CIMT and UAS4, duration of the disease, disease severity, CRP, MPV, NLR, PLR, LDL, total cholesterol and BMI in the patient group. However, a significant positive but inverse correlation was found between the right CIMT and the HDL value, and also a significant and positive correlation with TG value. In addition, there was a positive correlation between left CIMT and TG value (Table 3).

Table 1. Demographical data of the chronic spontaneous urticaria (CSU) and control groups

\begin{tabular}{|c|c|c|c|c|c|}
\hline \multicolumn{2}{|l|}{ Parameter } & \multirow{2}{*}{$\frac{\text { CSU }}{16(53.3)}$} & \multirow{2}{*}{$\begin{array}{c}\text { Healthy controls } \\
16(53.3)\end{array}$} & \multirow{2}{*}{$t$} & \multirow{2}{*}{$\begin{array}{c}P \text {-value } \\
-\end{array}$} \\
\hline Gender, $n(\%)$ & Female & & & & \\
\hline & Male & $14(46.7)$ & $14(46.7)$ & - & - \\
\hline \multicolumn{2}{|c|}{ Age, mean \pm SD [years] } & $37.7 \pm 7.7$ & $37.1 \pm 7.2$ & 0.52 & 0.959 \\
\hline \multicolumn{2}{|c|}{$\mathrm{BMI}$, mean $\pm \mathrm{SD}\left[\mathrm{kg} / \mathrm{m}^{2}\right]$} & $24.63 \pm 2.7$ & $24.83 \pm 2.5$ & 0.297 & 0.768 \\
\hline \multicolumn{2}{|c|}{ UAS, mean \pm SD } & $16.93 \pm 4.32$ & - & - & - \\
\hline \multicolumn{2}{|c|}{ Disease duration, mean \pm SD [months] } & $40.2 \pm 35.36$ & - & - & - \\
\hline
\end{tabular}

$B M I$ - body mass index.

Table 2. Laboratory findings and CIMT in the CSU and control groups

\begin{tabular}{|c|c|c|c|}
\hline Parameter & $\begin{array}{c}\text { CSU }(n=30) \\
\text { Mean } \pm \text { SD }\end{array}$ & $\begin{array}{l}\text { Healthy controls } \\
\qquad \begin{array}{l}(n=30) \\
\text { Mean } \pm \text { SD }\end{array}\end{array}$ & $P$-value \\
\hline $\mathrm{HDL}$ & $50.00 \pm 9.82$ & $59.70 \pm 12.60$ & $0.002^{*}$ \\
\hline Total cholesterol & $176.73 \pm 19.42$ & $169.70 \pm 26.82$ & 0.249 \\
\hline LDL & $100.13 \pm 16.80$ & $97.00 \pm 19.50$ & 0.508 \\
\hline TG & $101.07 \pm 33.87$ & $88.53 \pm 36.69$ & 0.174 \\
\hline CRP & $4.96 \pm 4.06$ & $0.80 \pm 0.65$ & $<0.0001^{*}$ \\
\hline MPV & $7.72 \pm 0.84$ & $8.39 \pm 2.16$ & 0.124 \\
\hline PLT & $268.2 \pm 62.19$ & $261 \pm 60.4$ & 0.689 \\
\hline NLO & $2.19 \pm 0.94$ & $2.91 \pm 5.00$ & 0.445 \\
\hline PLO & $124.13 \pm 44.19$ & $117.60 \pm 35.64$ & 0.530 \\
\hline Right CIMT & $0.52 \pm 0.09$ & $0.48 \pm 0.07$ & $0.043^{*}$ \\
\hline Left CIMT & $0.55 \pm 0.08$ & $0.50 \pm 0.07$ & $0.012^{*}$ \\
\hline
\end{tabular}

${ }^{*} P<0.05$ level of significance. CSU - chronic spontaneous urticaria, CRP - C-reactive protein, NLR-neutrophil lymphocyte ratio, HDL - high-density lipoprotein, $L D L$ - low-density lipoprotein, PLR - platelet lymphocyte ratio, TG - triglycerides, MPV-mean platelet volume, right CIMT - right carotid intima media thickness, left CIMT - left carotid intima media thickness. 
Table 3. Change of CIMT and CRP with disease duration, UAS, disease severity and laboratory findings

\begin{tabular}{lcccccc}
\hline Parameter & \multicolumn{2}{c}{ Right CIMT } & \multicolumn{2}{c}{ Left CIMT } & \multicolumn{2}{c}{ CRP } \\
\cline { 2 - 7 } & $R$ & $P$-value & $R$ & $P$-value & $R$ & $P$-value \\
\hline Total cholesterol & 0.275 & 0.142 & 0.141 & 0.457 & 0.00 & 0.998 \\
\hline BMI & 0.272 & 0.145 & 0.345 & 0.062 & 0.311 & 0.094 \\
\hline HDL & -0.484 & $0.007^{*}$ & -0.361 & $0.050^{*}$ & -0.460 & $0.010^{*}$ \\
\hline LDL & 0.498 & $0.005^{*}$ & 0.249 & 0.184 & 0.194 & 0.304 \\
\hline TG & 0.461 & $0.01^{*}$ & 0.498 & $0.05^{*}$ & 0.394 & $0.031^{*}$ \\
\hline CRP & 0.397 & $0.03^{*}$ & 0.427 & $0.019^{*}$ & - & - \\
\hline NLR & 0.0061 & 0.748 & 0.126 & 0.509 & 0.195 & 0.301 \\
\hline PLR & 0.038 & 0.842 & 0.050 & 0.794 & 0.129 & 0.498 \\
\hline UAS & 0.365 & $0.047^{*}$ & 0.417 & $0.022^{*}$ & 0.427 & $0.019^{*}$ \\
\hline Severity of disease & 0.436 & $0.016^{*}$ & 0.474 & $0.008^{*}$ & 0.486 & $0.006^{*}$ \\
\hline Disease duration & 0.245 & 0.192 & 0.083 & 0.662 & -0.123 & 0.516 \\
\hline *Corstion is sigifcat
\end{tabular}

${ }^{*}$ Correlation is significant at the 0.05 level. CRP - C-reactive protein, NLR-neutrophil lymphocyte ratio, HDL-high-density lipoprotein, LDL-low-density lipoprotein, PLR - platelet lymphocyte ratio, TG - triglycerides, BMI - body mass index, UAS - urticaria activity severity, right CIMT - right carotid intima media thickness, left CIMT - left carotid intima media thickness.

A statistically significant correlation was determined between CRP value and UAS and disease severity in the patient group. There was also a significant positive but inverse correlation between CRP and HDL values and a significant positive correlation with TG value. However, there was no correlation between duration of disease, MPV, NLR, PLR, LDL, and total cholesterol (Table 4).

There was no significant difference between the ASST positive and ASST negative patient groups in terms of CRP, NLR, PLR, MPV, total cholesterol, TG, HDL, LDL, right and left CIMT.

\section{Discussion}

We found that right and left CIMT values were statistically significantly higher in the CSU group as compared to the healthy control group and HDL values were significantly lower in the CSU patients as compared to the healthy control group and the CRP values were significantly higher in the CSU patient group. There was also a statistically significant positive correlation between CRP value and UAS, disease severity, and TG in the patient group, and a significant but inverse correlation with HDL. In addition, there was a significant but inverse correlation between right CIMT and HDL value, and a significant and positive correlation between right and left CIMT and TG value.

Chronic spontaneous urticaria is a persistent inflammatory disease of the skin characterized by non-necrotizing infiltration of CD4+ lymphocytes consisting of mast cell degranulation and perivascular Th1 and Th2 subtypes as well as monocytes, neutrophils, eosinophils and basophils [1], but there is little information currently available on the skin infiltration with such cells in CSU and the
Table 4. Laboratory findings and CIMT in ASST+ and ASST- patient group

\begin{tabular}{lccc}
\hline Parameter & $\begin{array}{c}\text { ASST+ } \\
(n=15) \\
\text { Mean } \pm \text { SD }\end{array}$ & $\begin{array}{c}\text { ASST - } \\
(n=15) \\
\text { Mean } \pm \text { SD }\end{array}$ & $P$-value \\
\hline HDL & $52.47 \pm 8.43$ & $47.53 \pm 10.75$ & 0.173 \\
\hline $\begin{array}{l}\text { Total } \\
\text { cholesterol }\end{array}$ & $178.2 \pm 16.77$ & $175.27 \pm 22.25$ & 0.687 \\
\hline LDL & $101.13 \pm 15.53$ & $99.13 \pm 18.47$ & 0.751 \\
\hline TG & $103.13 \pm 36.28$ & $99.00 \pm 32.42$ & 0.745 \\
\hline CRP & $5.73 \pm 3.74$ & $5.73 \pm 4.10$ & 0.995 \\
\hline MPV & $7.25 \pm 0.82$ & $7.60 \pm 1.02$ & 0.301 \\
\hline PLT & $284.60 \pm 84.420$ & $251.80 \pm 17.551$ & 0.152 \\
\hline NLO & $1.95 \pm 0.693$ & $2.44 \pm 1.11$ & 0.159 \\
\hline PLO & $128.10 \pm 49.37$ & $120.19 \pm 39.67$ & 0.632 \\
\hline Right CIMT & $0.54 \pm 0.07$ & $0.55 \pm 0.09$ & 0.893 \\
\hline Left CIMT & $0.51 \pm 0.09$ & $0.53 \pm 0.09$ & 0.672 \\
\hline ASST - Autolo & 55 S & & \\
\hline
\end{tabular}

ASST - Autologous Serum Skin Test, CRP - C-reactive protein, NLR - neutrophil lymphocyte ratio, HDL - high-density lipoprotein, $L D L$ - low-density lipoprotein, $P L R$ - platelet lymphocyte ratio, TG - triglycerides, $B M I$ - body mass index, UAS - urticaria activity severity, MPV - mean platelet volume, right CIMT - right carotid intima media thickness, left CIMT - left carotid intima media thickness.

warning that is responsible for that and systemic inflammatory response [1].

C-reactive protein is the most intensively studied inflammatory marker. C-reactive protein is an acute phase protein produced by main hepatocytes under the influence of cytokines such as TNF- $\alpha$ and IL-6 [20]. Danesh et al. [21] have found that even slight elevations in CRP concentrations between normal laboratory values may be associated with an increased cardiovascular risk. It has 
also been reported that the concentration of CRP increases in circulation in CSU patients [22]. Kasperska-Zajac et al. [22] have reported a positive correlation between CRP levels and urticaria severity and CRP levels, as well as the increase in CRP levels in CSU patients. Another study by Kasperska-Zając et al. [22] has reported that IL-6 and CRP levels were increased in CSU patients, and there is a correlation between IL-6, CRP and UAS. In addition, they have observed that IL- 6 and CRP level decreased in spontaneous remission [8]. In our study, we found that CRP levels were significantly increased in CSU patients in line with previous studies. We also observed a correlation between CRP levels and UAS and disease severity. These findings support the previous findings reporting that CSU showed low grade inflammatory symptoms $[1,8,22]$. It also supports that serum CRP concentration may be an objective parameter in evaluating disease severity in CSU.

During systemic inflammation, neutrophil activation and increased NLR reactive oxygen derivatives lead to the release of cytokines (IL-1RA, IL-6, IL-7, IL-8, IL-12), proteases and cationic proteins (such as elastase and lactoferrin) [6]. Neutrophil activation products have been shown to be associated with severity of various inflammatory diseases. It has been reported in recent years that neutrophils and associated lymphopenia are independent determinants of mortality in cardiovascular diseases and furthermore, NLR has been shown to be an inflammation indication in cardiac and non-cardiac diseases [3, 6, 23-25]. Karabay et al. [26] have reported significantly higher NLR values also in CSU patients as compared to healthy controls and also have found a positive correlation between serum CRP values and NLR values in CSU patients. However, they have observed no correlation between clinical disease severity and NLR value [26]. In our study, there was no significant difference in NLR values between the patient group with CSU and the control group, and there was no correlation between CRP and NLR values in the patient group.

The platelets interact with endothelial cells, leukocytes (monocytes, neutrophils, dendritic cells, T-cells) and progenitor cells in addition to their function of providing hemostasis, allowing the inflammatory cells to migrate to the lesion areas, causing abundant amounts of inflammatory cytokines to be released and eventually create an inflammatory environment in the lesion area. Platelets contribute to the formation of atherosclerotic plaques with surface receptors they express and the cytokines they secrete or to endothelial damage leading to thrombotic complications together with other components of inflammation [27]. It has been reported in recent years that MPV also reflects platelet function and activation and may be a marker of inflammation in different chronic diseases. It has also been shown that high PLR, as in NLR, is associated with mortality in cardiac diseases $[13,14$, 27]. We did not find a significant difference between the
MPV and PLR values in the CSU patient group compared to the healthy controls, and we found no significant correlation between MPV, PLR and CRP in the CSU patient group. We did not find any studies evaluating PLR in CSU in the literature. As far as we know, this is the first study to evaluate PLR in CSU. Similar MPV values determined in our study in CSU patients and in the healthy control group were similar to those of Zajac et al., but were not consistent with other studies that reported increases or decreases in MPV values [28-31].

Magen et al. [29] have reported in their study consisting of 373 patients with CSU and 46 healthy controls that increased MPV values might be an indicator of increased disease activity severity in patients with ASSTpositive CSU. Akelma et al. [31] have reported significantly lower MPV in pediatric patients compared to healthy controls.

These changes between studies may result from technical reasons and from different criteria used in the classification of patients. Especially in retrospective analyses, it may be due to failure to apply exclusion criteria including DM, HT, obesity, smoking, arterial and autoimmune disease. Lately, almost all diseases can increase or reduce MPV. Therefore, the practical possibility of excluding MPV-related diseases and conditions completely is difficult.

We did not find NLR, PLR and MPV values in CSU patients to be similar to those of healthy controls and not to be associated with CRP and disease severity in the CSU patient group. We therefore believe that MPV, NLR and PLR values are insufficient data for evaluating inflammation and disease severity in CSU. However, there is a need for more larger-scale prospective studies on the use of these parameters in CSU.

Studies show that skin diseases with chronic inflammation such as psoriasis and psoriatic arthritis pose a risk of atherosclerosis [19, 32]. Inflammation has an important role in the initiation and progression of atherosclerosis and circulatory diseases. Atherosclerosis is a chronic inflammatory and immunological disease in which many cells, including monocytes, macrophages, T lymphocytes, endothelial cells, smooth muscle cells and mast cells, are included [4]. Chronic spontaneous urticaria is thought to be a chronic inflammatory disease with cutaneous mast cell degranulation, T-cell, eosinophil, and neutrophil infiltration [1]. It has been shown that low-grade inflammation is associated with atherosclerosis and CSU. TNF- $\alpha$, IL-1-IL-2, IL-6, IL-8, IL-12, IL-18 are shown to be cytokines responsible for atherosclerotic plaque formation [4]. Increase in IL-6, TNF- $\alpha$, IL-1B, IL14, IL-13 and IL-18 levels were also observed in CSU patients [8-10, 33, 34]. Moreover, it is assumed that CSU is a nonallergic disease induced by activation of mast cells thought to be significantly associated with hyperlipidemia and atherosclerosis. Mast cells participate in both the natural and adaptive immune system and they play an important role in maintaining healthy physiology in wo- 
und healing angiogenesis. There is considerable evidence that mast cells play a key role in inflammation and that their products regulate inflammatory mediator production [35]. In recent years, mast cells have been shown in atherosclerotic plaque development and inflammatory disease processes such as cardiovascular disease, hyperlipidemia [36-38]. The mast cells present in the human arterial intima may be degranulated after stimulation and secrete the chymase inhibiting HDL apolipoprotein and delay cellular cholesterol flow. In fact, Lee et al. [39] have reported that mast cell chymase disrupts and decreases the level of APOA-1, the major HDL apolipoprotein that changes lipid metabolism.

In addition, the clinical effects and prevalence of metabolic syndrome among CSU patients was investigated in a hospital-based comparative cross-sectional study of 131 CSU patients and a higher prevalence of metabolic syndrome have been found in CSU patients [18]. They have reported that the presence of the metabolic syndrome in the logistic regression analysis correlated with a high urticaria activity score and that it was an independent risk factor for uncontrolled CSU. In another community-based cross-sectional study of 11261 CSU patients, the relationship between CSU and the metabolic syndrome has been investigated and in univariate analysis CSU has been found to be associated with higher metabolic syndrome and its components which are DM, obesity, hyperlipidemia and hypertension prevalences [40].

In recent studies, the measurement of intima media thickness with carotid artery USG, which is an inexpensive, simple and easily acceptable method for evaluating subclinical atherosclerosis, has been focused on [41]. Intima media thickness increase is considered as an atherosclerosis marker [15]. $0.1 \mathrm{~mm}$ increase in CIMT increases the myocardial infarction risk by $10-15 \%$ and the stroke risk by $13-18 \%$ [42].

In the light of this, it can be argued that when CSU is considered as an inflammatory disease, these patients may have an increased risk of atherosclerosis. In our study, we found that the values of right and left carotid intima media thickness were statistically significantly higher and the HDL value was significantly lower in CSU patients who did not have any known cardiovascular risk factor compared to the healthy control group. There was also an inverse correlation between CRP value and HDL and a positive correlation between these and TG in the CSU group. These findings support that there may be an increased risk of atherosclerosis in CSU patients. Our study is the first in the literature to investigate subclinical atherosclerosis in CSU patients. Having a small number of samples is the limiting factor of our study.

\section{Conclusions}

We are of the opinion that serum CRP concentration may be an objective parameter in evaluating disease se- verity in CSU but there are not sufficient data for the use of NLR, MPV and PLR in evaluating CSU inflammation and disease severity. On the other hand, we think that there is a need for larger-scale prospective studies on the use of these parameters in CSU. Furthermore, according to our results, impairment of lipid metabolism and atherosclerosis may be an increased trend in CSU patients, but more comprehensive prospective studies are needed to better define the relationship between the verification of these data and urticaria atherosclerosis.

\section{Conflict of interest}

The authors declare no conflict of interest.

\section{References}

1. Kasperska-Zajac A. Acute-phase response in chronic urticaria. J Eur Acad Dermatol Venereol 2012; 26: 665-72.

2. Zuberbier T, Aberer W, Asero R, et al.; European Academy of Allergy and Clinical Immunology; Global Allergy and Asthma EuropeanNetwork; European DermatologyForum; World Allergy Organization. The EAACI/GA(2)LEN/EDF/WAO guideline: for the definition, classification, diagnosis and management of urticaria: the 2013 revision and update. Allergy 2014; 69: 868-87.

3. Imtiaz F, Shafique K, Mirza SS, et al. Neutrophil to lymphocyte ratio as a measure of systemic inflammation in prevalent chronic diseases in Asian population. Int Arch Med 2012; 5: 2.

4. Conti P, Shaik-Dasthagırısaeb Y. Atherosclerosis: a chronic inflammatory disease mediated by mast cell. Cent Eur J Immunol 2015; 40: 380-6.

5. Kressel G, Trunz B, Bub A, et al. Systemic and vascular markers of inflammation in relation to metabolic syndrome and insulin resistance in adults with elevated atherosclerosis risk. Atherosclerosis 2009; 202: 263-71.

6. Coimbra S, Oliveira H, Reis F, et al. C-reactive protein and leucocyte activation in psoriasis vulgaris according to severity and therapy. J Eur Acad Dermatol Venereol 2010; 24: 789-96.

7. Nakanishi N, Sato M, Shirai K, et al. White blood cell count as a risk factor for hypertension; a study of Japanese male office workers. J Hypertens 2002; 20: 851-7.

8. Kasperska-Zajac A, Sztylc J, Machura E, Jop G. Plasma IL-6 concentration correlates with clinical disease activity and serum C-reactive protein concentration in chronic urticaria patients. Clin Exp Allergy 2011; 41: 1386-91.

9. Dos Santos JC, Azor MH, Nojima VY, et al. Increased circulating proinflammatory cytokines and imbalanced regulatory T-cell cytokines production in chronic idiopathic urticaria. Int Immunopharmacol 2008; 8: 1433-40.

10. Tedeschi A, Asero R, Lorini M, et al. Plasma levels of matrix metalloproteinase-9 in chronic urticaria patients correlate with disease severity and C-reactive protein but not with circulating histamine-releasing factors. Clin Exp Allergy 2010; 40: 875-81.

11. Buyukkaya E, Karakas MF, Karakas E, et al. Correlation of neutrophil to lymphocyte ratio with the presence and severity of metabolic syndrome. Clin Appl Thromb Hemost 2014; 20: 159-63. 
12. Hamminga EA, van der Lely AJ, Neumann HA, Thio HB. Chronic inflammation in psoriasis and obesity: implications for therapy. Med Hypotheses 2006; 67: 768-73.

13. Turkmen K, Erdur FM, Ozcicek F, et al. Platelet-to-lymphocyte ratio better predicts inflammation than neutrophil-to-lymphocyte ratio in end-stage renal disease patients. Hemodial Int 2013; 17: 391-6.

14. Canpolat F, Akpinar H, Eskioğlu F. Mean platelet volume in psoriasis and psoriatic arthritis. Clin Rheumatol 2010; 29 : 325-8.

15. Espeland MA, O'leary DH, Terry JG, et al. Carotid intimalmedia thickness as a surrogate for cardiovascular disease events in trials of HMG-CoA reductase inhibitors. Curr Control Trials Cardiovasc Med 2005; 6: 3.

16. Pignoli P, Tremoli E, Poli A, et al. Intimal plus medial thickness of the arterial wall: a direct measurement with ultrasound imaging. Circulation 1986; 74: 1399-406.

17. Coll B, Feinstein SB. Carotid intima-media thickness measurements: techniques and clinical relevance. Curr Atheroscler Rep 2008; 10: 444-50.

18. Ye YM, Jin HJ, Hwan EK, et al. Co-existence of chronic urticaria and metabolic syndrome: clinical implications. Acta Derm Venereol 2013; 93: 156-60.

19. Gisondi P, Fantin F, Del Giglio M, et al. Chronic plaque psoriasis is associated with increased arterial stiffness. Dermatology 2009; 218: 110-13.

20.Zacho J, Tybjaerg-Hansen A, Jensen JS, et al. Genetically elevated C-reactive protein and ischemic vascular disease. N Engl J Med 2008; 359: 1897-908.

21. Danesh J, Wheeler JG, Hirschfield GM, et al. C-reactive protein and other circulating markers of inflammation in the prediction of coronary heart disease. N Engl J Med 2004; 350: 1387-97.

22. Kasperska-Zajac A, Grzanka A, Machura E, et al. Analysis of procalcitonin and CRP concentrations in serum of patients with chronic spontaneous urticaria. Inflamm Res 2013; 62: 309-12.

23. Keizman D, Ish-Shalom M, Huang P, et al. The association of pretreatment neutrophil to lymphocyte ratio with response rate, progression free survival and overall survival of patients treated with sunitinib for metastatic renal cell carcinoma. Eur J Cancer 2012; 48: 202-8.

24. Sen N, Afsar B, Ozcan F, et al. The neutrophil to lymphocyte ratio was associated with impaired myocardial perfusion and long-term adverse outcome in patients with ST-elevated myocardial infarction undergoing primary coronary intervention. Atherosclerosis 2013; 228: 203-10.

25. Lee GK, Lee LC, Chong E, et al. The long-term predictive value of the neutrophil-to-lymphocyte ratio in type-2 diabetic patients presenting with acute myocardial infarction. QJM 2012: 105: 1075-82.

26. Karabay EA, Çerman AS, Altunay IK. Serum C-reactive protein, neutrophil-lymphocyte ratio and a uric acid levels in chronic spontaneous urticaria. Turkiye Klinikleri J Dermatol 2016; 26: 125-31.

27. Kasperska-Zajac A, Brzoza Z, Rogala B. Platelet function in cutaneous diseases. Platelets 2008; 19: 317-21.

28. Zajac AK, Grzanka A, Jarzab J, et al. The association between platelet count and acute phase response in chronic spontaneous urticaria. Biomed Res Int 2014; 2014: 650913.

29. Magen E, Mishal J, Zeldin Y, et al. Increased mean platelet volume and C-reactive protein levels in patients with chronic urticaria with a positive autologous serum skin test. Am J Med Sci 2010; 339: 504-8.
30. Magen E, Mishal J, Zeldin Y, Schlesinger M. Clinical and laboratory features of antihistamine-resistant chronic idiopathic urticaria. Allergy Asthma Proc 2011; 32: 460-6.

31. Akelma AZ, Mete E, Cizmeci MN, et al. The role of mean platelet volume as an inflammatory marker in children with chronic spontaneous urticaria. Allergol Immunopathol (Madr) 2015; 43: 10-3.

32. Tobin AM, Veale DJ, Fitzgerald O. Cardiovascular disease and risk factors in patients with psoriasis and psoriatic arthritis. J Rheumatol 2010; 37: 386-94.

33. Ferrer M, Luquin E, Sanchez-Ibarrola A, et al. Secretion of cytokines, histamine and leukotrienes in chronic urticaria. Int Arch Allergy Immunol 2002; 129: 254-60.

34. Caproni M, Cardinali C, Giomi B, et al. Serological detection of eotaxin, IL-4, IL-13, IFN-gamma, MIP-1alpha, TARC and IP10 in chronic autoimmune urticaria and chronic idiopathic urticaria. J Dermatol Sci 2004; 36: 57-9.

35. Fukuda S, Horiuchi S, Tomita K, et al. Acetylated low-density lipoprotein is endocytosed through coated pitsby rat peritoneal macrophages. Virchows Arch B Cell Pathol Incl Mol Pathol 1986; 52: 1-13.

36. Kritas SK, Saggini A, Varvara G, et al. Mast cell involvement in rheumatoid arthritis. J Biol Regul Homeost Agents 2013; 27: 655-60.

37. Artenjak A, Koželj M, Lakota K, et al. High avidity anti-beta2glycoprotein I antibodies activate human coronary artery endothelial cells and trigger peripheral blood mononuclear cell migration. Eur J Inflamm 2013; 11: 385-96.

38. Shaik-Dasthagirisaheb YB, Varvara G, Murmura G, et al. Vascular endothelial growth factor (VEGF), mastcells and inflammation. Int J Immunopathol Pharmacol 2013; 26: 327-35.

39. Lee M, Calabresi L, Chiesa G. Mast cell chymase degrades apoE and apoA-II in apoA-I-knockout mouse plasma and reduces its ability to promote cellular cholesterol efflux. Arterioscler Thromb Vasc Biol 2002; 22: 1475-81.

40.Shalom G, Magen E, Babaev M, et al. Chronic urticaria and the metabolic syndrome: a cross sectional community-based study of 11261 patients. JEADV 2018; 32: 276-81.

41. Eder L, Chandran V, Gladman DD. The Framingham Risk Score underestimates the extent of subclinical atherosclerosis in patients with psoriatic disease. Ann Rheum Dis 2014; 73: 1990-6.

42. Lorenz MW, Markus HS, Bots ML, et al. Prediction of clinical cardiovascular events with carotid intima-media thickness: a systematic review and meta-analysis. Circulation 2007; 115: 459-67. 